\title{
Mechanisms for the production of harmonics in free electron lasers
}

\author{
J.N. Elgin \\ Department of Mathematics, Imperial College, 180 Queen's Gate, London. SW7 2BZ. UK \\ C. Penman \\ Facultet der Technische Natuurkunde, Unversitett Twente, Postbus 217, 7500AE Enschede, Netherlands
}

\begin{abstract}
Harmonics in the radiation of a free electron laser are useful for extending the range of tuning, may originate in spontaneous or parametric processes, and can take part in stımulated emıssion or amplification. These mechanisms exhıbıt interestıng analogies with those of nonlınear optics. Apart from the well known nonparametric gain analogous to stımulated Brillouın scattering, they include third-harmonic generation and other four-wave mixing parametric processes, with spatial harmonics of the electron density takıng the place of the higher-order terms in the nonlınear dielectric susceptibility. We investigate these mechanisms using a one-dımensıonal model.
\end{abstract}

\section{Introduction: harmonics in spontaneous emission}

Harmonics in the incoherent radiation from an undulator appear as discrete peaks, more or less narrow depending on the number of periods, whose frequencies depend on the direction of observation. Their presence is due to the alteration of the form of periodic functions in the expression for the radiation field of a charge performing superımposed steady and periodic motions. These functions are evaluated at a retarded time $t^{\prime}$ given by the implicit function

$t^{\prime}(t)=t-R\left(t^{\prime}\right) / c$,

where $R$ is the distance from the source of the field to the point of observation. If $R$ contains a periodic part whose frequency is an integer multiple of $\Omega$, as happens in a planar undulator, then a periodic function, e.g. $\sin (\Omega t)$, composed with this retardation function becomes distorted as shown in fig. 1. This distortion produces the infinity of harmonics found with a plane undulator; a similar distortion, again due to a small periodic term in $R$, accounts for the off-axis harmonics of a helical undulator.

Because a plane undulator produces only odd harmonics, and a helical one none at all, on axis, the study of harmonics is usually connected with plane undulators. Even if harmonics are present they experience, according to the usual theory which ignores all powers of the radiation fields higher than the first, negligible gain on axis unless the undulator is of the planar type. The same restriction applies in the case of parametric processes due either to terms nonlinear in the radiation, or to the presence of a spatial component

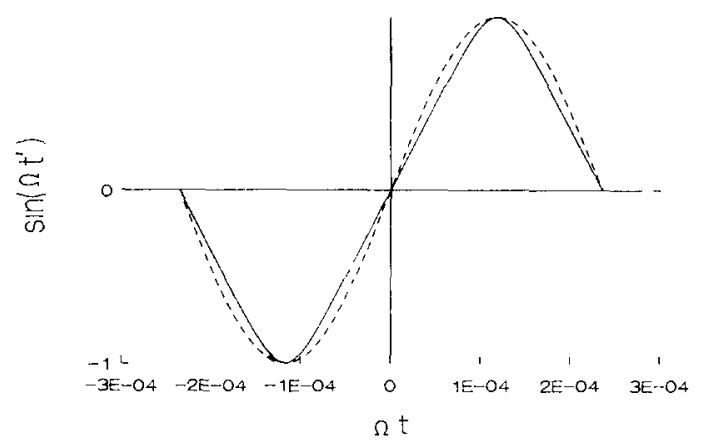

Fig. 1. Distortion of sine curve by planar-undulator retardation function: $\sin \left(\Omega t^{\prime}\right)$ vs $\Omega t$, with $t^{\prime}$ given by eq. $(1) .(\gamma=100$, $\kappa=1$; original curve is shown dotted).

in the electron density of the same harmonic number as the field component under consideration. We consider below some of these processes, in an attempt to demonstrate that the study of harmonics is worthwhile for the inherent interest of the mechanisms involved, and for the parallels which exist between these mechanisms and the processes of nonlinear optics, as well as for their use in extending the already large tuning range of the free electron laser.

\section{Harmonics in the coupled Maxwell-pendulum equa- tions}

We now consider various mechanisms apart from spontaneous emission for the production of harmonics on axis, which can be treated in a one-dimensional 
model. In a standard approach to the problem [1], the electron motion satisfies

$\frac{\mathrm{d} \gamma}{\mathrm{d} t}=\frac{-e^{2}}{\gamma m_{0}^{2} c^{2}} \boldsymbol{A} \cdot \boldsymbol{E}$,

where $\gamma$ is the electron relativistic factor, and $\boldsymbol{A}$ and $\boldsymbol{E}$ are, respectively, the vector potential and electric field strength of all fields, both electromagnetic and static. In the next step, $\boldsymbol{A}$ is usually approximated by the wiggler magnet contribution alone. Here we retain all contributions, from both radiation and wiggler fields. In this way, the following coupled evolution equations are obtained:

$$
\begin{aligned}
& \frac{\partial^{2} \zeta}{\partial Z^{2}}=\frac{1}{2} \sum_{p} a_{p} \mathrm{e}^{1 p \zeta}+\frac{\mathrm{i}}{2} \sum_{p, q} \frac{\chi_{p q}}{q} a_{p} a_{q} \mathrm{e}^{1(p+q) \zeta}, \\
& \frac{\partial a_{p}}{\partial Z}-\frac{1}{V} \frac{\partial a_{p}}{\partial \tau}=-J_{p}\left\langle\mathrm{e}^{-1 p \zeta}\right\rangle \\
& -\mathrm{i} j_{p} \sum_{q} \frac{\chi_{-p q}}{q} a_{q}\left\langle\mathrm{e}^{-1(p-q) 5}\right\rangle .
\end{aligned}
$$

Here $a_{p}=\left|a_{p}\right| \mathrm{e}^{1 \phi_{p}}$ is the complex amplitude of the $p$ th harmonic of the radiation field, with $p=1$ the fundamental mode; $Z$ is the propagation distance down the cavity, normalized to the undulator length $L$ (i.e. $Z=$ $z / L) ; \tau=(c t-z / \beta) / L$ is a retarded time variable, where $\beta c$ is the mean $z$-component of the electron velocity; $\zeta$ and $\nu$ are the canonical coordinate and momentum variables, respectively, with $\zeta=\left(k+k_{\mathrm{w}}\right) z$ $-\omega t$ the position (phase) of the electron within the potential well, and $\nu=\mathrm{d} \zeta / \mathrm{d} Z$ proportional to $\delta \gamma$, the energy "detuning" of the electron beam from its resonance value (defined by $\delta \gamma=0) ; 1 / V=(1 / \beta-1)$ is proportional to the slippage distance of the electron beam relative to the optical fields; and $J_{p}$ is a coupling constant related to linear gain. The normalization used is that due to Colson [1], and all canonical transformations required to obtain the variables $(\zeta, \nu ; Z)$ are described by Kroll et al. [2]. Note that $(\zeta, \nu)$ are microscopic variables, whereas $(Z, \tau)$ are macroscopic.

The sums in eqs. (3) and (4) cover both positive and negative values of $p$, with the convention $a_{-p} \equiv a_{p}^{*}$, $\phi_{-p} \equiv-\phi_{p}$. Only slowly varying contributions appear in eqs. (3) and (4); each $J_{p}$ therefore contains the familiar difference of Bessel functions $J_{(p-1) / 2}(p \xi)-$ $J_{(p+1) / 2}(p \xi)$, which is understood to apply for odd integers $p$ only. When $p$ is zero or even, $J_{p}=0$. The terms containing $\chi_{p q}, \chi_{-p q}$ arise from the radiation field contribution to the total vector potential $\boldsymbol{A}$. They are there because two radiation modes acting on the electron beam can bunch it in the same way as a single mode acting with the wiggler magnet.

It is easily found that

$\chi_{p q}=\frac{\kappa^{2}}{32 \pi^{2} N^{2}} g(p, q, \xi)$,

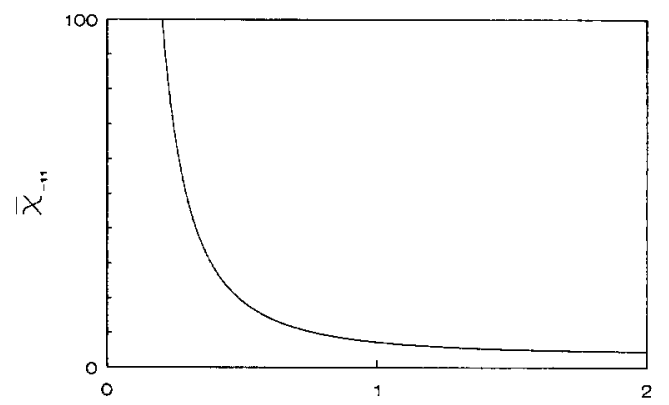

Fig. 2. $\bar{\chi}_{-1,1}$ vs $\kappa$ (eq. (5)).

where

$g(p, q, \xi)=(-1)^{(p+q) / 2} \frac{J_{(p+q) / 2}((p+q) \xi)}{\xi \kappa_{p}(\xi) \kappa_{q}(\xi)}$.

Here $\kappa=e A_{\mathrm{W}} /\left(m_{0} c\right)$, the normalized undulator field strength, and $\xi=\kappa^{2} /\left(4\left(1+\kappa^{2} / 2\right)\right)$. Also,

$\kappa_{p}=\kappa(-1)^{(p-1) / 2}\left(J_{(p-1) / 2}(p \xi)-J_{(p+1) / 2}(p \xi)\right)$,

as defined by Colson [1]. Finally, $N$ is the number of wiggler magnet periods. Since $\kappa$ is typically $\sim 1$, the constant factor in eq. (5) is very small, indicating that at all reasonable field strengths (i.e. small values of $\left|a_{p}\right|$ ) the contribution to harmonic generation from these terms is negligible. Plots of $\bar{\chi}_{p q} \equiv 32 \pi^{2} N^{2} \chi_{p q}$ are shown in figs. 2 and 3 for the cases $(p, q)=(-1,1)$ and $(3,1)$ respectively. In the special case when $p=-q$, we find

$J_{p} \chi_{p p}=\frac{k L}{2} \frac{\Omega^{2}}{\omega^{2}}$,

where $\Omega^{2}=\rho e^{2} /\left(\gamma \epsilon_{0} m_{0}\right)$ is the local plasma frequency of the electron beam. The resulting phase shift for the electromagnetic field is exactly that expected from propagation through a refractive medium with index $n$ such that $n^{2}=1-\Omega^{2} / \omega^{2}$.

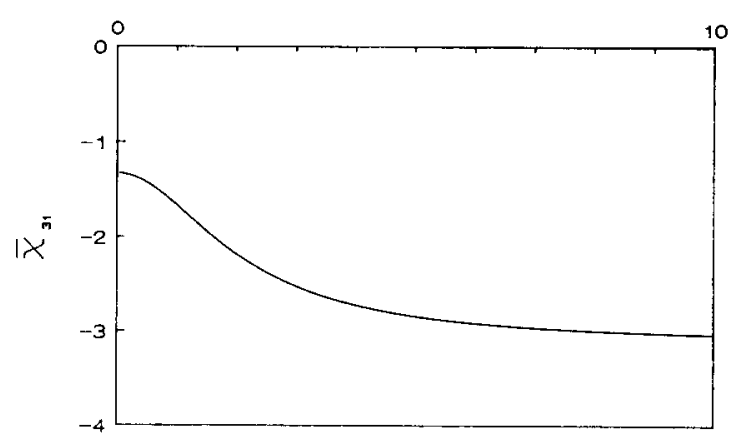

Fig. 3. $\bar{\chi}_{31}$ vs $\kappa$. 


\section{Results}

Consider now some special cases of the system of eqs. (3) and (4).

(i) With all $\chi$ set to zero, and a single harmonic field $a_{p}$ present, the situation is that discussed by Colson [1].

(i1) With all $\chi$ again set to zero, but now with a dominant fundamental mode bunching the electron beam, harmonic generation is again possible. The bunching at the fundamental wavelength is necessarily anharmonic, and hence has a nonzero Fourier coefficient at the $p$ th harmonic, represented by $\left\langle\mathrm{e}^{-1 p \zeta}\right\rangle$.

In practice, cases (i) and (1i) are present together and cannot be disentangled. However, they are physically different mechanisms: case (ii) is akın to parametric processes in nonlinear optics, whereas case (i) is essentially a nonparametric process. For the case when $p=3$, 1t can be shown that, for long pulses and for low gain, the second mechanism alone predicts [4]

$\frac{\partial a_{3}}{\partial Z}=\chi^{(3)} a_{1}^{3}$,

where

$$
\begin{aligned}
\chi^{(3)}= & \frac{1}{4} \frac{\kappa^{5}}{\kappa_{1}^{3}} \frac{J_{3}}{\nu_{0}^{6}}\left((0+\mathrm{i}(10)) \mathrm{e}_{0}\right. \\
& +\left(15 \nu_{0} Z+\mathrm{i}(-75)\right) \mathrm{e}_{1} \\
& +\left(-108 \nu_{0} Z+\mathrm{i}\left(-24\left(\nu_{0} Z\right)^{2}+150\right)\right) \mathrm{e}_{2} \\
& +\left(-18\left(\nu_{0} Z\right)^{3}+123 \nu_{0} Z\right. \\
& \left.\left.+1\left(72\left(\nu_{0} Z\right)^{2}-85\right)\right) \mathrm{e}_{3}\right) \\
\left(\mathrm{e}_{n}\right. & \left.=\exp \left(\mathrm{i}\left(3 \phi_{1}-n \nu_{0} Z\right)\right), \nu_{0}=\nu(z=0)\right) .
\end{aligned}
$$

Clearly, the mechanism is reminiscent of third-harmonic generation by a fundamental, with $\chi^{(3)}$ the third-order susceptibility of the electron beam. The growth in the third-harmonic amplitude is greatest for zero detuning,

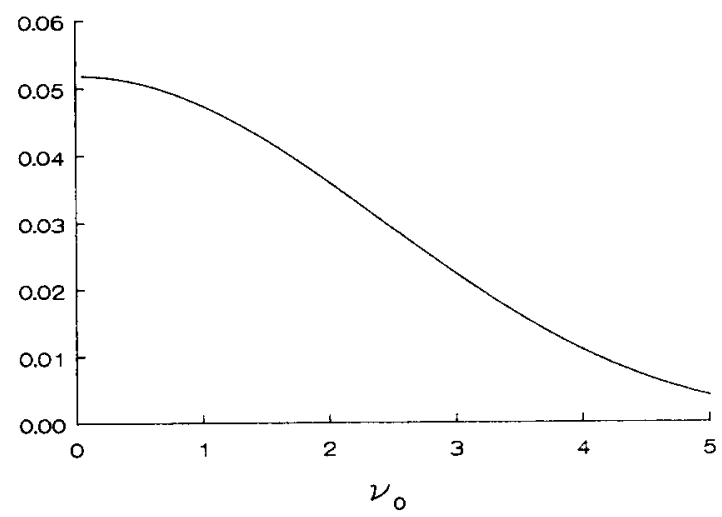

Fig. 4. $(1 / 1.08 / 3)\left(\kappa_{1}^{3} / \kappa^{5}\right)\left|\int_{0}^{1} \chi^{(3)}\left(v_{0} Z\right) \mathrm{d} Z\right|$ vs $\nu_{0}$ (eq. (10)). as can be seen from the plot in fig. 4 of a function of $\nu_{0}$ proportional to $\left|\int_{0}^{1} \chi^{(3)}\left(\nu_{0} Z\right) \mathrm{d} Z\right|$ (the constant being chosen to relate the integrated thurd-harmonic field in Colson's normalization [1] to the small-sıgnal gain $(0.27$ $\left.\left(\kappa / \kappa_{p}\right)^{2} j_{p}\right)$.

Consider now the cases when $\chi \neq 0$.

(iii) Continuing with the special case of third-harmonic generation, and setting $p=3, q=1 \mathrm{in}$ eq. (4) an additional source term is revealed:

$-\mathrm{i} J_{3} \times_{31} a_{1}\left\langle\mathrm{e}^{-21 \xi}\right\rangle$.

That is, bunching at the second harmonic couples with the fundamental mode to drive the third harmonic. This mechanism has recently been examined by Bonifacio et al. [3], who argue that for high-gain systems it can be the dominant mechanism.

Finally, we illustrate an example where the system of eqs. (3) and (4) behaves rather like an optical parametric oscillator: setting $p= \pm 1, q=\mp 1$ in eq. (4), respectively, we find

$$
\begin{aligned}
& \frac{\partial a}{\partial z}-\frac{1}{V} \frac{\partial a}{\partial \tau}=-J\left\langle\mathrm{e}^{-1 \zeta}\right\rangle+\mathrm{i}_{J} \chi_{11} a^{*}\left\langle\mathrm{e}^{-21 \zeta}\right\rangle, \\
& \frac{\partial a^{*}}{\partial z}-\frac{1}{V} \frac{\partial a^{*}}{\partial \tau}=-J\left\langle\mathrm{e}^{1 \zeta}\right\rangle-{ }_{1} J \chi_{11} a\left\langle\mathrm{e}^{21 \zeta}\right\rangle .
\end{aligned}
$$

The last terms in each equation cross-couple the equations, in the manner of an optical parametric oscillator. Each is driven by a bunching at the second harmonic, which plays the part of the pump mode. If the terms in $\mathrm{e}^{-15}$ are zero, as at resonance, there may still be growth in the fundamental from the nonlinear terms. Subharmonics may also be generated by a similar process; the generating equations would then be a parametric pair coupling, e.g. $a_{1 / 2}, a_{1 / 2}^{*}$, through bunching at the fundamental.

\section{Conclusion}

Not surprisingly, the FEL is an interesting source of nonlinear phenomena. To date the simplest of such processes has received most attention - where a single harmonic bunches the electron beam and is in turn generated by the bunched beam - but this process is only one of the many worthy of further study.

\section{References}

[1] W.B Colson, IEEE J. Quantum Electron. QE-17 (1981) 1417

[2] N M. Kroll, P L. Morton and M.N. Rosenbluth, IEEE J Quantum Electron. QE-17 (1981) 1436

[3] R. Bonifacio, L. De Salvo and P. Pierıni, Nucl. Instr. and Meth. A239 (1990) 36.

[4] C Penman, Ph.D. Thesis, Department of Mathematics, Imperial College, London (1988). 